\title{
Switch-Independent Task Representations in Frontal and Parietal Cortex
}

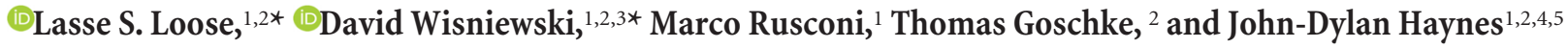 \\ ${ }^{1}$ Bernstein Center for Computational Neuroscience Berlin and Berlin Center for Advanced Neuroimaging, Charité Universitätsmedizin Berlin, 10115 Berlin, \\ Germany, ${ }^{2}$ Department of Psychology and Collaborative Research Center Volition and Cognitive Control, Technische Universität Dresden, 01069 Dresden, \\ Germany, ${ }^{3}$ Department of Experimental Psychology, Ghent University, Ghent, Belgium, ${ }^{4}$ Cluster of Excellence NeuroCure, Charité, and ${ }^{5}$ Humboldt- \\ Universität zu Berlin, Berlin School of Mind and Brain and Institute of Psychology, 10099 Berlin, Germany
}

Alternating between two tasks is effortful and impairs performance. Previous fMRI studies have found increased activity in frontoparietal cortex when task switching is required. One possibility is that the additional control demands for switch trials are met by strengthening task representations in the human brain. Alternatively, on switch trials, the residual representation of the previous task might impede the buildup of a neural task representation. This would predict weaker task representations on switch trials, thus also explaining the performance costs. To test this, male and female participants were cued to perform one of two similar tasks, with the task being repeated or switched between successive trials. Multivoxel pattern analysis was used to test which regions encode the tasks and whether this encoding differs between switch and repeat trials. As expected, we found information about task representations in frontal and parietal cortex, but there was no difference in the decoding accuracy of task-related information between switch and repeat trials. Using crossclassification, we found that the frontoparietal cortex encodes tasks using a generalizable spatial pattern in switch and repeat trials. Therefore, task representations in frontal and parietal cortex are largely switch independent. We found no evidence that neural information about task representations in these regions can explain behavioral costs usually associated with task switching.

Key words: cognitive control; fMRI; MVPA; parietal cortex; task switching; task set

\section{Significance Statement}

Alternating between two tasks is effortful and slows down performance. One possible explanation is that the representations in the human brain need time to build up and are thus weaker on switch trials, explaining performance costs. Alternatively, task representations might even be enhanced to overcome the previous task. Here, we used a combination of fMRI and a brain classifier to test whether the additional control demands under switching conditions lead to an increased or decreased strength of task representations in frontoparietal brain regions. We found that task representations are not modulated significantly by switching processes and generalize across switching conditions. Therefore, task representations in the human brain cannot account for the performance costs associated with alternating between tasks.

\section{Introduction}

To reach desired goals, humans are often required to switch between different tasks. This important aspect of cognitive control

\footnotetext{
Received Nov. 23, 2016; revised May 15, 2017; accepted June 21, 2017.

Author contributions: L.S.L., D.W., M.R., T.G., and J.-D.H. designed research; L.S.L. and D.W. performed research; L.S.L. and D.W. analyzed data; L.S.L., D.W., T.G., and J.-D.H. wrote the paper.

This work was supported by the Bernstein Computational Neuroscience Program of the German Federal Ministry of Education and Research (Grant 01GQ1001C); the German Research Foundation within the Collaborative Research Center Volition and Cognitive Control: Mechanisms, Modulations, Dysfunctions (DFG Grants SFB 940/1 and SFB 940/2); German Research Foundation Grants Exc 257, Neurocure, and KF0247. This research was also supported by the Research Foundation Flanders (FW0) and the European Union's Horizon 2020 research and innovation programme under the Marie Skłodowska-Curie grant agreement No. 665501, and FW0 grant FW0.0PR.2013.0136.01. We thank Kai Görgen, Achim Meier, Fernando Ramírez, Jelle Demanet, and Marcel Brass for helpful comments on this project. The authors declare no competing financial interests.

*L.S.L. and D.W. contributed equally to this work.
}

allows flexible adjustment of behavior to changing circumstances (Kok et al., 2006). Such adjustments are often investigated using the task-switching paradigm, requiring subjects to switch frequently between two or more tasks (Meiran, 2010). Typically, participants react more slowly and perform less accurately on tasks that they just switched to compared with tasks that were repeated multiple times (Jersild, 1927; Spector and Biederman, 1976). These switch costs (Rogers and Monsell, 1995) reflect cognitive control processes (Goschke, 2000) that affect task processing and the implementation of tasks (Monsell, 2003), as well as

Correspondence should be addressed to Lasse $S$. Loose, Bernstein Center for Computational Neuroscience, Humboldt-Universität zu Berlin, Philippstr. 13, Haus 6, 10115 Berlin. E-mail: lasse.loose@bccn-berlin.de. DOI:10.1523/JNEUROSCI.3656-16.2017

Copyright $\odot 2017$ the authors $\quad 0270-6474 / 17 / 378033-10 \$ 15.00 / 0$ 
A Trial structure

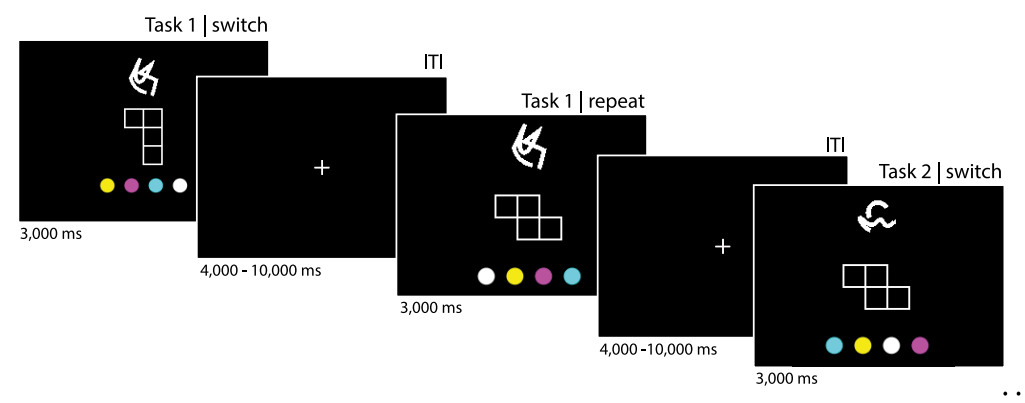

B Stimulus-response associations \& task cues

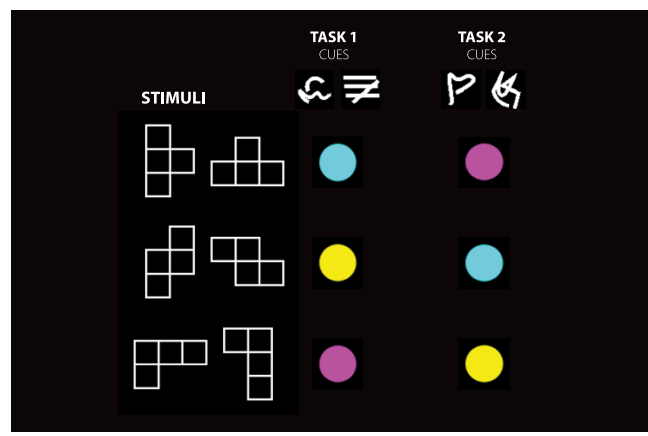

Figure 1. Experimental paradigm. A, Trial structure. Each trial consisted of a target stimulus at fixation, a cue stimulus above, and four possible response options below. Each task screen was presented for $3000 \mathrm{~ms}$, during which time participants could indicate a response using the left and right index and middle fingers. Each trial was followed by a fixation cross with a variable ITI $(4000-10,000 \mathrm{~ms}$, mean $=5525 \mathrm{~ms})$. Responses were indicated by pressing the button corresponding to the mapped color on screen on a $2 \times 2$ button box with their index and middle fingers of both hands. Subjects were cued to perform one of two tasks, switching between tasks or repeating a task up to three consecutive times. B, S-R associations and task cues. The two tasks consisted of similar stimulus response mappings, associating stimulus shapes (in two possible orientations) with colors. Each task was indicated by one of two possible abstract cues.

proactive interference and between-task crosstalk (Allport et al., 1994; Yeung et al., 2006). However, the exact sources of switch costs are still under debate (Kiesel et al., 2010).

Previous fMRI studies investigated the neural basis of preparatory processes in task switching using univariate methods (Ruge et al., 2013). Although many results implicate the prefrontal and parietal cortical regions in task switching (Dove et al., 2000; Gruber et al., 2006; Jamadar et al., 2010), this finding is not always consistent (Ruge et al., 2013). Previous task-switching research mostly focused on neural correlates of task-switching processes in terms of BOLD signal differences between switch and repeat trials. Recently, multivoxel pattern analysis (MVPA) (Haynes, 2015) has been used to investigate neural task representations. Such representations are encoded in local spatial activation patterns in the lateral prefrontal, dorsal anterior cingulate, and posterior parietal cortex (Bode and Haynes, 2009; Gilbert, 2011; Woolgar et al., 2011; Wisniewski et al., 2015).

Different cognitive processes such as rule complexity (Woolgar et al., 2015) or skill acquisition (Jimura et al., 2014) have been shown to alter representations of tasks. However, whether and how task switching (and its associated cognitive control demands) influences task representations is still largely unknown. Behavioral switch costs in task switching reflect both the cognitive control processes required to switch to performing a different task, as well as involuntary processes such as proactive interference from a previous task set (Kiesel et al., 2010; Vandierendonck et al., 2010). Possibly, this also affects the representation of these tasks (Waskom et al., 2014). In other cases, task representations remain unaffected by whether tasks were chosen freely or were externally cued (Wisniewski et al., 2016) or if tasks were novel or were performed routinely (Cole et al., 2011). This suggests that tasks can also be represented independently of current cognitive control demands (Zhang et al., 2013). However, it has remained open whether and how task-switch related control demands and between-task crosstalk in task-switching contexts affect the neural representation of tasks.

To investigate the influence of task switching on task representations, two main questions are addressed in this study. First, do different cognitive control demands on task-switch versus task-repeat trials affect the strength of neural tasks representations? More specifically, does the accuracy with which tasks can be decoded from neural activation patterns differ between taskswitch and task-repeat trials? Second, is the neural code in which tasks are represented independent of control demands? Therefore, are tasks encoded using generalizable spatial activation patterns in switch and repeat trials?

To address these questions, subjects were instructed to perform one of two simple stimulus-response (S-R) mapping tasks while brain activity was measured with fMRI. We identified brain networks involved in representing tasks and then assessed task information in these regions for switch versus repeat trials separately. Furthermore, we tested whether brain regions encode tasks invariant to switch and repeat condition. Results indicated that tasks are represented generalizably in a frontoparietal network, suggesting that switch-related cognitive control demands exert no strong effect on neural task representations.

\section{Materials and Methods \\ Participants}

Forty-two right-handed subjects (21 females, 21 males, mean age: 25.2 years, range 20-30 years) with normal or corrected-to-normal vision participated in the study. We obtained written informed consent from each subject and the local ethics committee approved the experiment. Subjects received $30 €$ for their participation. No subject had a selfreported history of neurological or psychiatric disorders. We only invited subjects to the fMRI session whose accuracy in performing the tasks after training was $>90 \%$. Therefore, we had to discard one subject after the training session because of poor behavioral performance (see experimental paradigm). We discarded two further subjects because of technical problems during scanning and one subject due to excessive head movement during scanning. To ensure reliable behavioral performance, all subjects took part in a training session $1-3 \mathrm{~d}$ before the scanning. Overall, the fMRI data of 38 subjects ( 20 females, mean age: 25 , age range 20-29 years) were used for our analyses.

\section{Task and experimental paradigm}

Subjects were cued to apply one of two S-R mappings (tasks) to a visual stimulus in each trial of the experiment. In half of the trials, the task was identical to the previous trial (repeat trials); in the other half of the trials, the task differed from the previous trial (switch trials). We instructed subjects to respond as quickly and accurately as possible.

The experiment was programmed using MATLAB version 7.11.0 (The MathWorks, RRID: SCR_001622) and the Cogent Toolbox (http://www. vislab.ucl.ac.uk/cogent.php). On each trial, we first presented a task screen for $3000 \mathrm{~ms}$ that simultaneously displayed a task cue, a target stimulus, and four colored circles used for the response-mapping assignment (Fig. 1 and see below). Subjects were allowed to respond in the same 3000 ms time window. The task screen was followed by an intertrial 
interval (ITI) during which a fixation cross was presented centrally on the screen. ITIs varied among 4000, 6000, 8000, and 10,000 ms and were distributed pseudologarithmically to decorrelate trials in time. The mean ITI was $5525 \mathrm{~ms}$.

Tasks were cued using abstract visual symbols presented at the top of the screen. They were free of semantic meaning to avoid a priori semantic associations (Fig. 1; Reverberi et al., 2012a; Wisniewski et al., 2015). Over the experiment, two different cues were associated with each task to allow for cue-independent task decoding (see below for details; also see Reverberi et al., 2012a, b). The cue-task associations were counterbalanced across subjects. The target stimuli consisted of three geometric objects (Fig. 1, T-shape, L-shape, Z-shape), each appearing in two possible orientations ( 0 and 90 degrees) and presented in the middle of the screen. Stimuli and their orientations were pseudorandomized to control for the influence of low-level visual features. The two tasks consisted of different S-R mappings that associated stimulus shapes with colors that in turn specified which response key had to be pressed. In task one, the T-shaped stimulus was associated with magenta, the Z-shaped stimulus with cyan, and the L-shaped stimulus with yellow. In task two, the T-shaped stimulus was associated with cyan, the Z-shaped stimulus with yellow, and the $\mathrm{L}$-shaped stimulus with magenta. The S-R mappings were chosen to be similar to control for possible confounds due to difficulty differences between tasks (Todd et al., 2013). Furthermore, switch costs can also be affected by task difficulty (Arbuthnott, 2008). Below the target stimulus, four colored circles were presented that mapped colors to response buttons. The position of the each colored circle was pseudorandomized across trials, avoiding motor preparation of responses as well as balancing left-hand and right-hand button presses throughout the experiment. Subjects used index and middle fingers of both hands to indicate their response by pressing the button corresponding to the color on screen on a $2 \times 2$ button box (Current Designs). Three of the circles were relevant for the task (cyan, magenta, and yellow) and one was a dummy (white) that was not relevant in any trial. This was done to balance left and right button presses.

Each run contained 80 trials, which were ordered so that $50 \%$ appeared in a sequence length of 1 (e.g., task 1 ), $37.5 \%$ in a sequence length of 2 (e.g., task 1 - task 1), and $12.5 \%$ in a sequence length of 3 (e.g., task $1-$ task $1-$ task 1 ). This resulted in 50\% switch trials and 50\% repeat trials overall. In $50 \%$ of the trials, subjects performed task 1 and, in the other $50 \%$, they performed task 2 . Tasks and switch conditions were orthogonalized. Within each brief sequence of identical tasks, we only used one of the two possible cues; that is, in each of the subsequent repetitions of a task, the same cue was used (cue repetition). Please note that such cue repetitions might confound the measured switch cost because it might be composed in parts of or simply reflect cue-switch cost only. Given that we focused our analyses on the representation of task, not on the switch processes directly, this does not affect the interpretation of our main results (Mayr and Kliegl, 2003; but see Altmann, 2006, 2007). Furthermore, cues were counterbalanced with stimuli and ITIs to avoid possible confounds.

Following each completed run, the percentage of correct answered trials was presented and subjects were offered a short resting break of self-determined length. Subjects performed five runs in total. The experiment lasted $\sim 75 \mathrm{~min}$ in total. A sixth run, in which subjects performed the tasks in a different sequential order, was not analyzed and is not included here.

One to $3 \mathrm{~d}$ before the scanning session, subjects performed a $90 \mathrm{~min}$ training session, during which they learned the S-R mappings. At the end of the training session, they performed two runs of the task as they would be presented in the scanner. Only if the accuracy during these runs was $>90 \%$ were subjects invited to the scanning session. This was done to avoid possible learning effects during the MRI session.

\section{Image acquisition}

Functional imaging was conducted on a $3 \mathrm{~T}$ Siemens Trio scanner equipped with a 12-channel head coil. For each of the five relevant scanning sessions, we acquired $347 \mathrm{~T} 2^{*}$-weighted $(\mathrm{TR}=2000 \mathrm{~ms}$; TE, $30 \mathrm{~ms}$; flip angle, $90^{\circ}$ ) gradient-echo echoplanar images (EPI). Imaging parameters were as follows: TR, $2000 \mathrm{~ms}$; TE, $30 \mathrm{~ms}$; and flip angle, $90^{\circ}$. Each volume contained 33 slices (thickness: $3 \mathrm{~mm}$ ) separated by gaps of 0.75 $\mathrm{mm}$. Matrix size was $64 \times 64$, the field of view (FOV) was $192 \mathrm{~mm}$, and the in-plane voxel resolution was set to $3 \mathrm{~mm}^{2}$ with a voxel size of $3 \times$ $3 \times 3 \mathrm{~mm}$. A T1-weighted structural dataset was also collected. The parameters were as follows: TR, $1900 \mathrm{~ms}$; TE, $2.52 \mathrm{~ms}$; matrix size, $256 \times$ 256; FOV, $256 \mathrm{~mm}$; 192 slices ( $1 \mathrm{mmt}$ thick); flip angle, $9^{\circ}$.

\section{Statistical analysis}

In all analyses, only trials with correct responses and preceded by correct trials (no misses/errors) were included to avoid post-error slowing effects (Dudschig and Jentzsch, 2009). We analyzed behavioral and fMRI data using MATLAB version 2013a (The MathWorks). For the multivariate analyses, we used The Decoding Toolbox (TDT; Hebart et al., 2016). Unthresholded group-level parametric maps of all analyses can be found at NeuroVault (Gorgolewski et al., 2016, RRID: SCR_003806; http://neurovault.org/collections/2011/).

\section{Behavior}

For each subject, we assessed task performance by calculating the mean reaction time (RT) and mean accuracy (i.e., the percentage of trials that were correctly answered in time) across all runs. It has been reported previously that switching between tasks leads to increased RT and decreased accuracy in switch trials compared with repeat trials (Monsell, 2003). We tested these so-called switch costs by comparing switch and repeat trials in terms of mean RT and accuracy using paired-sample, one-sided $t$ tests. To control for possible influences of task difficulty, we also assessed the influence of the two tasks and the four cues on RTs and accuracies using paired $t$ tests and one-way repeated-measures ANOVAs, respectively. We expected task switches to have an effect on both accuracy and RT (switch cost), but did not expect the other variables to affect them.

\section{Neuroimaging}

First-level GLM analysis

In a first step, we analyzed functional data using SPM8 (http://www.fil. ion.ucl.ac.uk/spm, RRID: SCR_007037). The functional volumes were unwarped, realigned, and slice time corrected. No spatial smoothing and no spatial normalization was applied at this point to preserve finegrained patterns of voxel activations (Haynes and Rees, 2006).

The preprocessed data were used to estimate a voxelwise general linear model (GLM; Friston et al., 1994). Twelve regressors of interest were used in the GLM. First, regressors for the eight conditions of the experimental design: two (tasks) $\times$ two (cue-sets) $\times$ two (switch/repeat) were added. Second, four separate regressors of no interest were added, modeling the four possible button presses to control for possible motor confounds in the data. Third, movement parameters were added to the GLM as regressors of no interest to account for possible head movement during scanning. Regressors were time locked to the onset of the task screen and convolved with a canonical hemodynamic response function as implemented in SPM.

To account for the possible influence of task difficulty on MVPA results (Todd et al., 2013), we first calculated the mean RT for task 1 and task 2 for each subjects individually. We then set the duration of each regressor to the mean task RT of the current trial (mean RT task 1 for trials with task 1 and mean RT task 2 for trials with task 2 , as suggested by Woolgar et al., 2014). This accounts for task-specific RT-related effects in the data during GLM estimation, but does not remove task-switchrelated variance from the data (Vandierendonck et al., 2010; for recent reviews about switch cost, see Kiesel et al., 2010).

\section{Multivariate searchlight decoding}

Analysis 1: Differences in task coding in switch and repeat trials. To test for possible differences of task representations in switch and repeat trials, we first identified regions that code for tasks and, in the following steps, assessed the differences of task-decoding in switch and repeat trials separately in these regions. Our analyses were designed to test whether task information is encoded similarly in switch and repeat trials. Put differently, can task information be read out in a similar way in switch and repeat trials? This type of question can be very well addressed using linear 
classifiers (Kriegeskorte, 2011), which we used in every decoding analysis presented here.

Analysis 1A: Task information across all trials. In the first analysis, we used "searchlight" MVPA (Kriegeskorte et al., 2006; Norman et al., 2006) as implemented in TDT (Hebart et al., 2016) on the maps of GLM parameter estimates for each individual subject. For each voxel $\mathrm{V}$ in the volume, the searchlight classifier distinguishes between the two classes (here: tasks) based on the multivariate pattern formed by the local fMRI activity patterns in a small spherical cluster with the radius of 3 voxels surrounding V. We used a support vector classifier (SVC) with a linear kernel and a fixed regularization parameter $(\mathrm{C}=1)$ as implemented in LIBSVM (http://www.csie.ntu.edu.tw/ cjlin/libsvm). As a result, searchlight decoding produces a whole-brain accuracy map representing which searchlights contained information about the two classes entered into the analysis. To identify which brain regions contain information about tasks, we performed this first searchlight decoding analysis, classifying task 1 versus task 2 and using data from both switch and repeat trials combined. Trials were collapsed across switch and repeat condition to increase power to identify regions of interests (ROIs) that contain information about tasks. To control for the effect of visual cue information, we performed cross-classification across visual cues. More specifically, we trained the SVC to discriminate "task 1 with cue 1 " and "task 2 with cue 2 " and tested its performance on trials from "task 1 with cue 3 " and "task 2 with cue 4 ." Only brain regions that use similar activation patterns to encode the same tasks with different cues will be visible in this analysis. Therefore, this analysis controls for effects that are merely due to the visual features of the cues used. There are a total of four different combinations of task and visual cues as a training and test dataset, so that we repeated this analysis three more times, once for every combination. To address the problem of overfitting (Kriegeskorte et al., 2009), we performed a fivefold leave-one-run-out cross-validation. Therefore, every run was the test dataset once. The results of the combinations of crossvalidation and cross-classification were averaged for each subject.

The average accuracy maps were then spatially normalized to a standard brain (Montreal Neurological Institute EPI template of SPM8) to account for individual differences in brain structure. Accuracy maps were then smoothed with a Gaussian kernel $(6 \mathrm{~mm}$ full-width at halfmaximum) to account for differences in localization. At the group level, a random-effects analysis was performed using voxelwise one-sample $t$ tests against chance level (50\%). Results were initially thresholded at voxel level with $p<0.001$, corrected for multiple comparisons at the cluster level for familywise error (FWE) $(p<0.05)$. Note that these threshold values are not problematic for cluster-level inference regarding the inflated FEW rates that have been discovered recently by Eklund et al. (2016).

Analysis 1B: Differences in task decoding for switch and repeat trials. In a second step, we performed two additional searchlight decoding analyses that were highly similar to Analysis 1A described above. This time, we performed two independent analyses for switch trials only and repeat trials only. We first entered only the data of switch trials into a SVC that was trained to classify task 1 versus task 2 . We again applied crossclassification across cues and leave-one-run-out cross-validation and averaged across them. We also smoothed and normalized the resulting decoding accuracy maps, as described above. The same procedure was repeated for repeat trials only. This yielded a task-decoding accuracy map for switch trials and for repeat trials for each individual subject. To compare the task-decoding accuracies in switch and repeat trials, we created ROIs from the clusters that we defined in task decoding Analysis 1A. To avoid circular analysis (Kriegeskorte et al., 2009), we used a leave-onesubject-out ROI analysis (Esterman et al., 2010). For this, we excluded one subject and performed a group-level analysis as described above (Analysis 1A). The results were then thresholded at voxel level with $p<$ 0.001 (corrected for multiple comparisons at the cluster level, FWE, $p<$ 0.05 ). We extracted the resulting significant clusters from this analysis and created a ROI from each cluster (based only on the training subjects). For each ROI thus defined, we extracted the mean decoding accuracy for the left-out subject. The ROI should resemble the group level results of Analysis 1A, but ensure an independent dataset for extracting decoding accuracies. Accuracy values were extracted for the decoding of task in switch trials only, repeat trials only, and all trials together (Analysis 1A). We repeated this procedure until every subject was left out once. This ensures independence of the data used to define the ROIs from the data used for statistical assessment of the accuracy values inside these ROIs. The mean decoding accuracies from all three analyses and all ROIs were then entered into a two-factorial repeated-measures ANOVA (factor 1: 3 analyses; factor 2: ROIs) to identify possible differences between task coding in switch and repeat trials in each ROI. Furthermore, to assess whether decoding accuracies were significantly above chance in each analysis and ROI, planned one-tailed $t$ test against chance level were performed. Results from these tests were Bonferroni corrected for the three analyses performed in each ROI.

To complement results of the traditional $t$ tests, we also calculated Bayes factors (BFs) using R (RStudio version 1.0.136; RRID: SCR_001905, package: BayesFactor). Classical null hypothesis significance testing comes with several limitations, one of them being that these tests do not provide evidence for the null hypothesis (Wagenmakers, 2007; Dienes, 2014). Following these methods, the absence of a significant effect does not provide information whether there was an absence of an effect in the data or if the data were inconclusive in this regard. Bayesian hypothesis testing enables us to quantify evidence in favor for the null hypothesis (Rouder et al., 2009; Mertens and De Houwer, 2016). Following Jeffreys (1961), we considered BFs between $1 / 3$ and 1 to represent anecdotal and thus inconclusive evidence and BFs smaller than $1 / 3$ and $1 / 10$ to indicate substantial and strong evidence for the absence of an effect, respectively. $\mathrm{BFs}$ larger than 1 were considered to represent substantial and BFs larger than 3 to represent strong evidence for the presence of an effect.

Analysis 2: Generalization of task coding between switch and repeat trials. Please note that the abovementioned analysis (1B) merely tests whether brain regions that encode tasks have different accuracies in switch and in repeat trials. If a given ROI indeed has a higher accuracy in one or the other condition, this would indicate a specialized role for task coding in either switch or repeat trials. If, however, no difference were to be found, this would not show directly that the ROI has a similar role in switch and repeat trials. To assess directly whether any brain regions encode tasks switching independently in these two conditions, a different type of analysis is necessary. Therefore, in Analysis 2, we aimed to identify brain regions that encode task information in the same way independently of whether subjects were repeating or switching between tasks, again using cross-classification (Reverberi et al., 2012a; Kaplan et al., 2015; Wisniewski et al., 2016). Similar to Analysis 1A, we first trained a searchlight classifier to distinguish between tasks in switch trials only and tested it on repeat trials only. We then trained a classifier on repeat trials only and tested it on switch trials only. Again, in both cases, we used leave-onerun-out cross-validation to avoid the problem of overfitting. Results from both analyses were first averaged for both cross-classification directions and then smoothed and normalized as in the previous analyses.

Please note that, in contrast to the Analysis 1, this analysis does not control for the effect of visual features of the task cues and results might reflect these. We also performed an exploratory task-decoding analysis with cross-classification across visual cue and across switch/repeat conditions at the same time. Specifically, we trained the classifier on "task 1 switch trials with cue 1" versus "task 2 switch trials with cue 3 " and tested on "task 1 repeat trials with cue 2 " versus "task 2 repeat trials with cue 4 ." Such combined cross-classification should reveal task representations that are independent of switch and not confounded by visual cue information. This additional division of training data allows for 12 possible combinations of training and testing datasets. Therefore, we repeated this analysis for each combination and also applied cross-validation as described in the analyses above. The resulting decoding maps were averaged for each subject. However, because the trial number used to train the classifier in each decoding is again split in half compared with the previous cross-classifications, data are now divided across the cue and switch condition. That reduces the power of this analysis considerably, likely leading to reduced decoding accuracies.

To still control for the effect of visual cue information, we again used the ROIs defined in Analysis 1A using the leave-one-subject-out method. Within these clusters, we now extracted the mean task-decoding accuracies from Analysis 2, where we cross-classified across switch and repeat 
trials. Please note that this is similar to a conjunction analysis and only voxels that show significant above-chance information in both task decoding cross-classified across visual cues and task decoding crossclassified across the switch and repeat conditions are interpreted. If tasks are encoded generalizably in these regions, then the mean decoding accuracies of task in both analyses should be significantly above chance. We tested this by applying a $t$ test (against chance level, 50\%) on the mean decoding accuracies for each cluster.

\section{Results}

\section{Behavior}

The mean RT across all correct trials was $1681 \mathrm{~ms}(\mathrm{SE}=30 \mathrm{~ms})$. After removing trials following error trials, the mean RT changed significantly to $1664 \mathrm{~ms}\left(\mathrm{SE}=27 \mathrm{~ms}\right.$; paired $t$ test: $t_{(37)}=3.69$; $p<0.001 ; \mathrm{BF}=42$ ). This effect could reflect post-error slowing (Dudschig and Jentzsch, 2009). All fMRI and RT analyses are based only on correct trials also following a correct trial. On average, subjects were correct and fast enough in 95.5\% (SE = $0.6 \%)$ of the trials. In $2.9 \%$ of the trials $(\mathrm{SE}=0.3 \%)$ subjects pressed the wrong button and, in $1.6 \%$ ( $\mathrm{SE}=0.3 \%$ ), they did not respond within the $3000 \mathrm{~ms}$ response window. The mean RT did not differ significantly between the two tasks (paired $t$ test, $t_{(37)}=$ $-0.30, p=0.76 ; \mathrm{BF}=0.18$ ), nor did the accuracy of both tasks. Furthermore, there was no significant effect of cue on RTs, as tested using a one-way repeated measures $\operatorname{ANOVA}\left(F_{(3,37)}=0.31\right.$, $p=0.81 ; \mathrm{BF}=0.05)$. No effects of tasks (paired $t$ test, $t_{(37)}=0.47$, $p=0.74 ; \mathrm{BF}=0.22$ ) or cues (ANOVA, $F_{(3,37)}=1.17, p=0.32 ; \mathrm{BF}=$ 0.13 ) were found in accuracy rates. The average $\mathrm{RT}$ in switch trials was $1699 \mathrm{~ms}(\mathrm{SE}=32 \mathrm{~ms})$. The average $\mathrm{RT}$ in repetition trials was $1656 \mathrm{~ms}$ ( $\mathrm{SE}=30 \mathrm{~ms})$. The difference between these switch and repeat trials (switch cost) was significant (paired $t$ test, $t_{(37)}=$ 5.04; $p<0.001 ; \mathrm{BF}=1645)$. Average accuracy in switch trials was $94.59 \%(\mathrm{SE}=0.68 \%)$ and, in repeat trials, $96.36 \%(\mathrm{SE}=0.5 \%)$. This difference was also significant (paired $t$ test, $t_{(37)}=-4.44$; $p<0.001 ; \mathrm{BF}=308)$. These results replicate previous findings of switch cost in RT and accuracy values (Monsell, 2003).

\section{Multivariate searchlight decoding}

Analysis 1: Differences in task coding in switch and repeat trials Analysis 1A: Task information across all trials. First, we identified regions that encode tasks using data from both switch and repeat trials combined. Using cross-classification, we ensured that the visual features of the task cues cannot explain the results. Significant above-chance classification of task could be observed in three clusters $(p<0.05$, FWE corrected at the cluster level, initial voxel threshold $p<0.001$; Fig. $2 A$, Table 1$)$. The first cluster is located in left inferior and superior parietal cortex spanning across angular gyrus, the second in right superior parietal cortex spanning across angular gyrus, and the third in left prefrontal cortex (PFC).

Analysis 1B: Differences in task decoding for switch and repeat trials. To compare the task-decoding accuracies in switch-only and repeat-only trials, we used a leave-one-subject-out approach to create the ROIs from the clusters identified in Analysis 1A. This procedure avoids the problem of double dipping (Kriegeskorte et al., 2009). We then extracted the task-decoding accuracy values in switch-only and repeat-only conditions. Figure $2 B$ shows an overlay of all leave-one-subject-out-ROIs that were created. As expected, they closely resemble task-decoding results across all subjects in Analysis 1A. A two-factorial repeated-measures ANOVA on the mean task-decoding accuracies in these ROIs showed no significant main effect of the decoding analysis (all/ switch-only/repeat-only task decodings, $F_{(2,74)}=0.06, p=0.94$; $\mathrm{BF}<0.001)$, no significant main effect of the ROI $\left(F_{(2,74)}=0.59\right.$, $p=0.55 ; \mathrm{BF}<0.001)$, and no interaction effect between ROI and the decoding analysis $\left(F_{(4,148)}=1.08, p=0.36 ; \mathrm{BF}=0.06\right)$. This indicates that there are no strong differences in the task-decoding accuracies between switch and repeat trials in task-related brain regions. We thus have strong evidence for the absence of an effect in this analysis.

Average task decoding in the left parietal cortex in all trials was $52.44 \%(\mathrm{SE}=0.64 \%)$, which was significantly above chance level $\left(50 \%, t\right.$ test: $\left.t_{(37)}=3.82 ; p<0.001 ; \mathrm{BF}=60\right)$. In switch trials only, the average decoding accuracy was $52.46 \%(\mathrm{SE}=0.79 \%)$ and, in repeat trials only, it was $51.83 \%$ ( $\mathrm{SE}=0.89 \%)$. In right parietal cortex, the task-decoding accuracy in all trials was $52.1 \%$ ( $\mathrm{SE}=$ $0.54 \%$ ) and was significantly above chance level $\left(t\right.$ test: $t_{(37)}=$ 3.87; $p<0.001 ; \mathrm{BF}=66)$. In switch trials only, it was $52.05 \%$ $(\mathrm{SE}=0.69 \%)$ and, in repeat trials only, it was $51.75 \%(\mathrm{SE}=$ 0.76). In left lateral PFC, the task-decoding accuracy in all trials was $51.85 \%(\mathrm{SE}=0.5 \%)$ and was significantly above chance level $\left(t\right.$ test: $\left.t_{(37)}=3.733 ; p<0.001 ; \mathrm{BF}=46\right)$. In switch trials only, it was $51.35 \%(\mathrm{SE}=0.6 \%)$ and, in repeat trials only, it was $52.18 \%$ $(\mathrm{SE}=0.83 \%)$.

Please note that, due to the physical limitations of fMRI, we cannot exclude that additional information is present at finer spatial scales. The absence of differences between task-decoding accuracies in switch and repeat trials is thus limited by the spatial resolution of fMRI.

Although this is not the focus of our study, we also performed a univariate contrast of switch-repeat and repeat—switch trials and a multivariate decoding of switch versus repeat trials. Neither the univariate contrasts nor the multivariate decoding of switch versus repeat yielded significant results at $p<0.05$ FWE cluster correction and initial voxel threshold of $p<0.001$. The univariate results are consistent with previous research (Cavina-Pratesi et al., 2006; Gruber et al., 2006), although results on this topic have been heterogeneous (Ruge et al., 2013).

\section{Analysis 2: Generalization of task coding between switch and repeat trials}

In Analysis 1, we did not find evidence for a difference in task coding between switch and repeat trials. In a next step, we assessed directly whether regions that encode task do so invariant to switch and repeat condition. We thus performed a task-decoding analysis, training on switch trials and testing on repeat trials. To ensure an independent test dataset, we again used the ROIs extracted from Analysis 1A using a leave-one-subject-out approach. We extracted the mean decoding accuracy in these ROIs from the task-decoding analysis cross-classified across the switch/ repeat conditions. Mean decoding of task was significantly above chance level $(50 \%)$ in the left parietal $\left(t\right.$ test: $t_{(37)}=4.84 ; p<$ $0.001 ; \mathrm{BF}=940)$, right parietal $\left(t\right.$ test: $t_{(37)}=5.05 ; p<0.001$; $\mathrm{BF}=1719)$, and left prefrontal $\left(t\right.$ test: $t_{(37)}=2.83 ; p<0.001$; $\mathrm{BF}=5.31)$ regions. We thus have strong evidence for the presence of an effect in this analysis. This finding indicates that all identified task-related brain regions encode tasks generalizably regardless of the current switch/repeat condition.

To assess whether any other regions outside of the ROIs investigated above also encode tasks similarly invariant to switch and repeat conditions, we performed an additional explorative whole-brain analysis of the task decoding using cross-classification across switch/repeat trials.

Results were thresholded at voxel level with $p<0.001$, corrected for multiple comparisons at the cluster level (FWE, $p<$ $0.05)$. Task information was found in bilateral inferior and superior parietal cortex, bilateral precuneus, right angular gyrus, and 

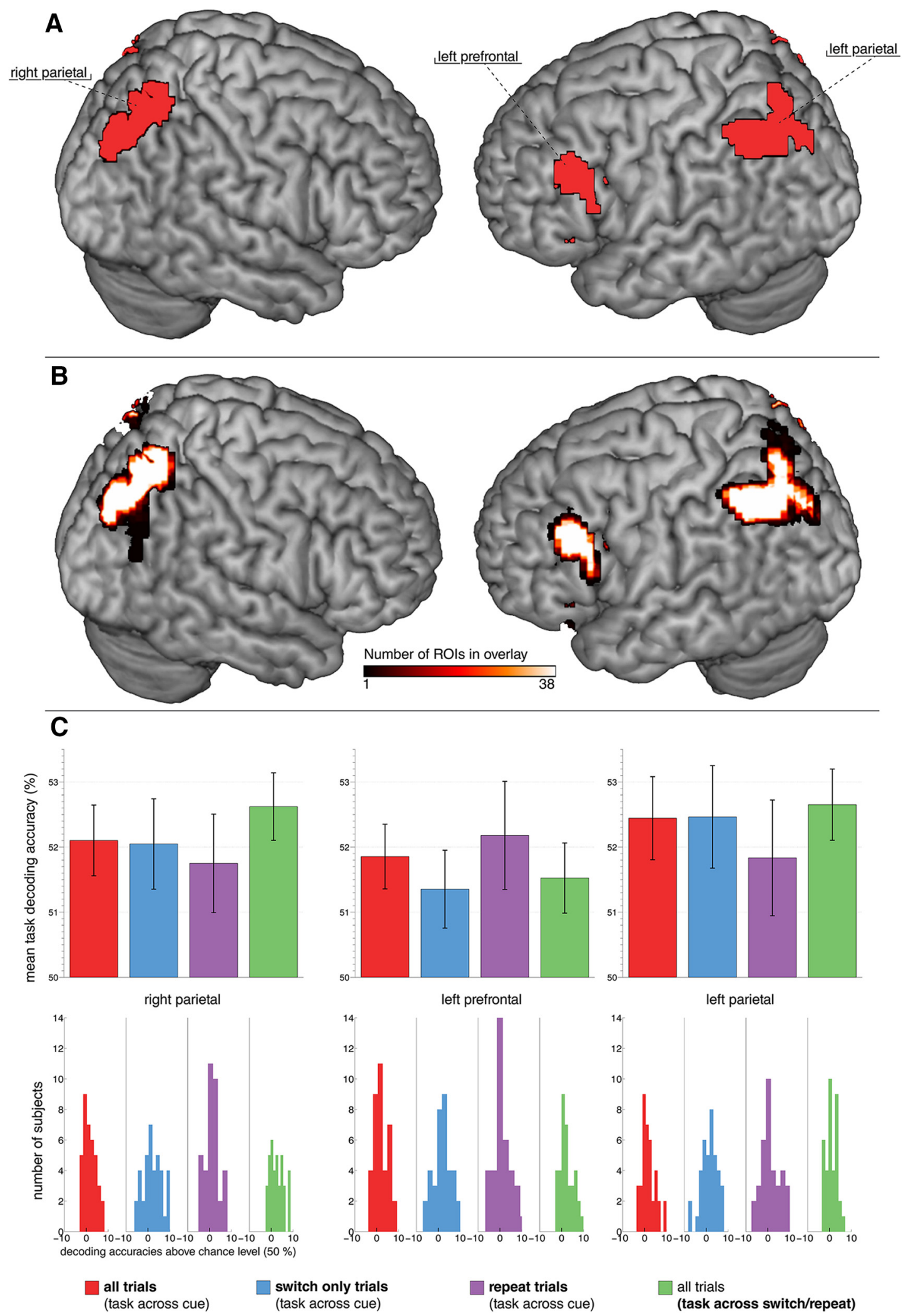

Figure 2. Task decoding. $A$, Task decoding across cues in all trials and all subjects. Tasks were encoded in bilateral superior parietal cortex, left inferior parietal cortex, and left lateral PFC $(p<0.05$, FWE corrected at the cluster level, initial voxel threshold $p<0.001)$. B, Overlay of all 38 leave-one-subject-out ROls. All ROls were created leaving out one subject at the group-level statistic $(p<0.05$, FWE corrected at the cluster level, initial voxel threshold $p<0.001)$ and later used for extraction of mean decoding accuracy values in that subject. $\boldsymbol{C}$, Mean task-decoding accuracies extracted from the Rols depicted in $\boldsymbol{B}$. We extracted values from four different decodings: task across cues in all trials (red), task across cues in switch trials only (blue), task across cues in repeat trials only (violet), and task across switch (green). Chance level in these plots is 50\%. The distribution of mean decoding accuracies across subjects is shown in the histograms below. 
Table 1. Results of analysis

\begin{tabular}{|c|c|c|c|c|c|c|}
\hline \multirow[b]{2}{*}{ Brain region } & \multirow[b]{2}{*}{ Side } & \multirow{2}{*}{$\begin{array}{l}\text { Cluster } \\
\text { size }\end{array}$} & \multicolumn{3}{|c|}{$\begin{array}{l}\text { MNI coordinates } \\
\text { (peak voxels) }\end{array}$} & \multirow{2}{*}{$\begin{array}{l}\text { tscore } \\
\text { peak }\end{array}$} \\
\hline & & & $x$ & $y$ & $z$ & \\
\hline \multicolumn{7}{|l|}{ Task across cues in all trials ${ }^{a}$} \\
\hline Parietal lobe & Left & 383 & -51 & -52 & 49 & 4.75 \\
\hline Parietal lobe & Right & 293 & 36 & -61 & 64 & 4.87 \\
\hline Prefrontal lobe & Right & 261 & -39 & 35 & -2 & 5.51 \\
\hline \multicolumn{7}{|l|}{ Task across switch in all trials ${ }^{b}$} \\
\hline Parietal lobe & Left & 1053 & -48 & -55 & 49 & 5.17 \\
\hline Parietal lobe & Right & 597 & 27 & -64 & 46 & 5.71 \\
\hline Occipital lobe & Left & 440 & -21 & -91 & -2 & 4.69 \\
\hline Cerebellum & Left & 173 & -39 & -64 & -23 & 5.34 \\
\hline Occipital lobe, cerebellum & Right & 135 & 45 & -64 & -11 & 4.8 \\
\hline
\end{tabular}

${ }^{a}$ Brain regions where tasks could be decoded in an analysis collapsed across switch and stay trials independently of visual cue.

${ }^{b}$ Brain regions where classifiers trained on switch trials could be used to decode the task in repeat trials and vice versa.

bilateral occipital cortex spanning into bilateral cerebellum (Fig. 3 , green). Please note that, in contrast to Analysis 1, this analysis does not control for the effect of visual features of the task cues and results might reflect these. We therefore performed a conjunction analysis with the regions identified in Analysis 1A. This analysis explicitly controls for the influence of visual cue features on task decoding results. Voxels found in both Analysis 1A and Analysis 2 thus encode tasks in a generalizable format invariant to different visual cues and different switch/repeat conditions. This conjunction analysis identified the bilateral parietal cortex (Fig. 3 , yellow). In contrast to Analysis 1A, we did not find a prefrontal cluster. Please note that this whole-brain analysis is less sensitive than our leave-one-subject-out ROI approach, potentially explaining the absence of prefrontal findings. This analysis suggests that the task information in parietal cortex generalizes across multiple different contexts.

To further test whether tasks are encoded in a switchindependent fashion, we compared the accuracies of task decoding within and across switching conditions directly. If a task representation is encoded independently of the switch/repeat condition, then it should yield comparable decoding accuracies in decoding in which the classifier is trained on switch and also tested on switch trials and the classifier is trained on switch but tested on repeat trials. For this comparison, we averaged the individual subjects' task-across-cue-decodings for switch only and repeat only trials from Analysis 1B and compared them with the task decoding across cue and across switch conditions simultaneously. Please note that the number of trials used to train the classifier for all of these analyses is the same in both analyses. Decoding accuracies did not differ significantly in left parietal (paired $t$ test: $t_{(37)}=-0.8730, p=0.3883, \mathrm{BF}=0.25$ ), right parietal (paired $t$ test: $t_{(37)}=-0.9427, p=0.3520, \mathrm{BF}=0.26$ ), or left prefrontal (paired $t$ test: $t_{(37)}=-1.1629, p=0.2523, \mathrm{BF}=$ $0.32)$ cortex.

The task decoding across cue and across switch condition simultaneously did not yield any significant results at FEWcorrected cluster level $p<0.05$ with initial voxel threshold $p<$ 0.001 . This is likely due to the reduced number of trials that were used to train the classifier.

To further corroborate our findings, we repeated all analyses using correlation-based classifiers as implemented in TDT (Hebart et al., 2016). We compared the whole-brain results of the analyses above (linear SVC) with respective exploratory correlation-based classifier results using a post hoc paired $t$ test as implemented in SPM8. Results did not differ significantly on a whole-brain level (initial voxel threshold $p<0.001$, FWE-corrected FWE cluster level $p<0.05)$. To test whether the classifier type had an influence on the results identified in more sensitive ROI analyses, we performed an additional ANOVA on the ROI results. We repeated the ANOVA described above, including the factors ROI (left parietal, right parietal, left prefrontal) and decoding analysis (task across cue for all trials, switch only trials, and repeat only trials). We added a third factor, classifier type, to this ANOVA (linear support vector classification, correlation-based classification). Results indicated that the main effect of classifier type did not reach significance $\left(F_{(1,37)}=1.95, p=0.17, \mathrm{BF}<0.001\right)$.This ANOVA was designed to be as similar as possible to the original ANOVA performed in Analysis 1B. Because this did not include the decodings under Analysis 2, we assessed the influence of classifier on these analyses with two separate ANOVA, including the factors of classifier type and ROI (as described above). However, we found no significant effect of classifier type in either of the remaining analyses: task across switch condition $\left(F_{(1,37)}=0.14\right.$, $p=0.71, \mathrm{BF}<0.001)$ and task across cue and switch condition $\left(F_{(1,37)}=1.62, p=0.21, \mathrm{BF}<0.001\right)$. Overall, we have strong evidence for the absence of any differences between the two classification algorithms used here, showing that our results are not specific to the method that we used.

\section{Discussion}

\section{Summary}

Effective goal-directed behavior requires humans to switch frequently between different tasks. To direct this behavior, cognitive control is required (Kok et al., 2006). Much previous research used task-switching paradigms to examine the role of cognitive control when changing between tasks (Monsell, 2003; Kiesel et al., 2010). Results show that performance is modulated by switching and switch costs are observed in both RT and accuracy (Jersild, 1927; Allport et al., 1994). However, the cognitive mechanisms and neuronal correlates of this behavioral switch cost are still under debate (Kiesel et al., 2010). Most previous fMRI research has focused on the neural correlates of task-switching processes (Ruge et al., 2013) and task-switching-related processes have been associated with a frontoparietal control network (Sohn et al., 2000; Braver et al., 2003; Brass et al., 2005; Crone et al., 2006). However, most of this research focused on the processes required to reconfigure the cognitive system from performing one task to performing a different task. Presumably, this includes changes to the neural representations of tasks, but effects on neural task representations have rarely been investigated (but see Waskom et al., 2014). However, task representations have been shown to be context dependent in some cases (Woolgar et al., 2015) and to remain context independent in others (Wisniewski et al., 2016). Here, we investigated the influence of cognitive control processes related to task switching on the neural representations of tasks.

In the current study, subjects were cued to perform one of two simple tasks, with the task being repeated or switched between successive trials. Behavioral results indicate that subjects showed switch costs (Rogers and Monsell, 1995), which suggests that cognitive control demands differed between switch and repeat trials. We first compared task-decoding accuracies in switch and repeat trials in these regions. Our results show that tasks were represented in bilateral parietal cortex and left lateral PFC. However, we found no differences in task-decoding accuracies between switch and repeat trials. Therefore, our data yielded no evidence that tasks are represented differently for either switch or repeat trials in the regions that we identified previously to maintain task information (but see Waskom et al., 2014). We also 


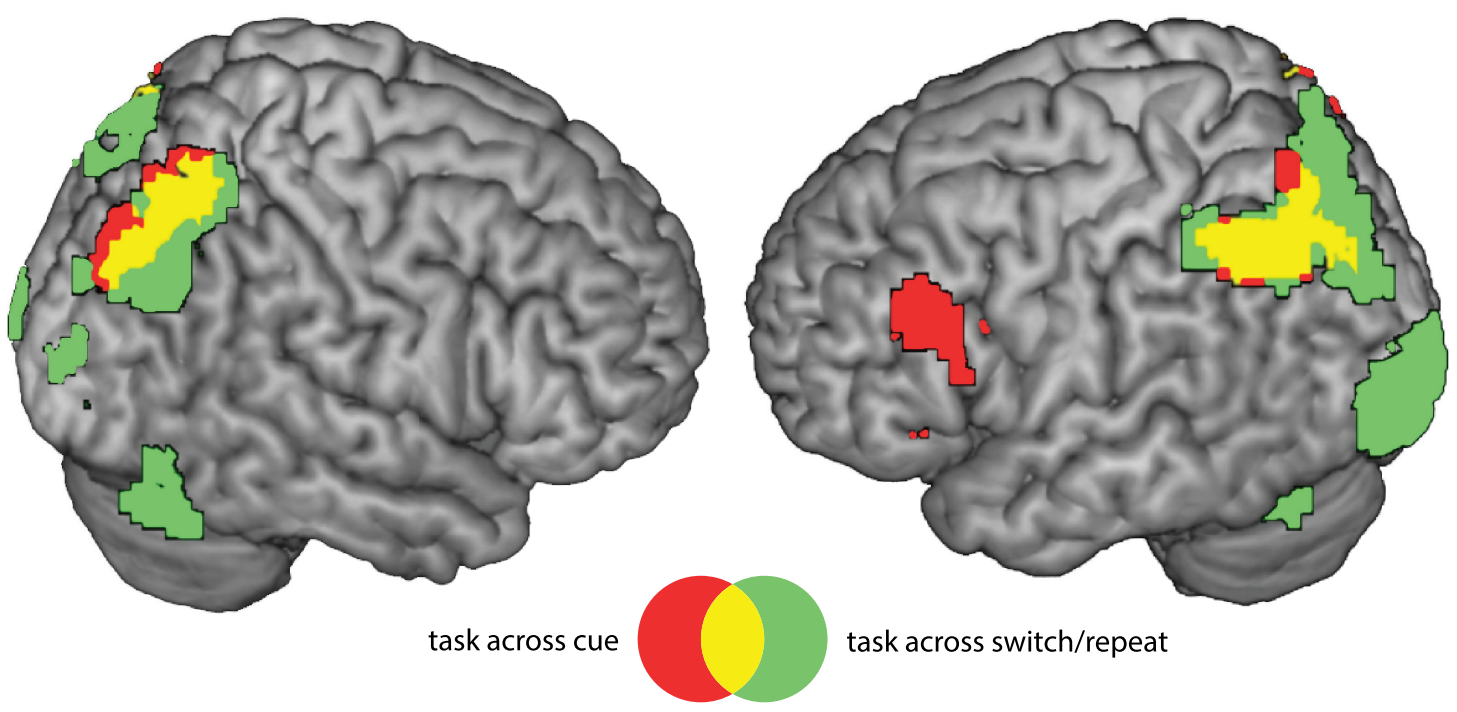

Figure 3. Task decoding in all subjects for the decoding of task across cues (red, Analysis 1A) and the decoding of task across switch/repeat (green, Analysis 2). Both regions overlap Note that task decoding across switch/repeat does not control for the visual information contained in task cues (which might explain occipital task information) and is also less sensitive than the leave-one-subjectROl approach, which might account for no prefrontal cluster surviving cluster correction (for both analyses: $p<0.05$, FWE corrected at the cluster level, voxel threshold $p<0.001$ ).

tested for generalization of task coding across switch and repeat trials using cross-classification. Results indicate that the frontoparietal cortex represents tasks regardless of the current cognitive control demands in task switching and suggests that tasks are coded in a robust, switching-independent pattern.

\section{Task representations in frontoparietal cortex}

Recent MVPA research investigating the neural representations of tasks directly has shown that parietal (Bode and Haynes, 2009; Woolgar et al., 2011; Waskom et al., 2014; Etzel et al., 2016; Wisniewski et al., 2015), medial (Gilbert, 2011; Momennejad and Haynes, 2013), and lateral PFC (Cole et al., 2011; Reverberi et al., 2012 b) hold information about tasks. We provide further evidence for these findings because we were able to discriminate between the two highly similar tasks in bilateral parietal and left lateral PFC. This is consistent with previous results highlighting the important role of these regions in task processing during task retrieval and maintenance (Bunge et al., 2003; Sakai and Passingham, 2003; Gilbert, 2011), processing rule and task compositionality (Woolgar et al., 2011; Reverberi et al., 2012a), adaptively coding tasks under different conditions (Woolgar et al., 2011), and their engagement over the course of development (Wendelken et al., 2012).

\section{Influence of switching on task representation in frontoparietal cortex}

Recent studies suggest that task representations can be modulated by different contextual variables: task representations have been observed to be modulated by rule complexity (Woolgar et al., 2015), rewards (Etzel et al., 2016), and skill acquisition (Jimura et al., 2014). This illustrates how higher cognitive functions might change flexibly the way task are processed in the brain, possibly reflecting adaptation of neuronal populations to different environmental demands (Duncan, 2010, 2013). However, other studies suggest that task representations also remain unaffected by experimental manipulation, such as task novelty (Cole et al., 2011), task difficulty (Wisniewski et al., 2015), or whether they are freely chosen or externally cued (Zhang et al., 2013; Wisniewski et al., 2016). It remains an open question whether and how cognitive control processes modulate task representations. In a previous study, Waskom et al. (2014) found task information in the inferior frontal and intraparietal sulcus, as well as the occipitotemporal cortex. They found representations of rules regarding perceptual discriminations to be modulated by task switching because they had the highest decoding accuracy after a task switch. Such effects on context information might be driven by attentional processes (Liu and Hou, 2013). Also note that Waskom et al. (2014) did not observe behavioral switch costs. It thus remains unclear whether cognitive control demands differed between switch and repeat trials and if these neuroimaging results in fact reflect control-related processes. In contrast, our subjects did show switch costs, indicating different control demands between switch and repeat trials. Importantly, because we presented task cues simultaneously with the task stimuli, participants could not prepare in advance for the new task on switch trials. Therefore, switch costs presumably reflect both effects of task set inertia and proactive interference, as well as increased control demands due to the requirement to retrieve and implement the new task set and to reconfigure S-R accordingly. Nevertheless, our results suggest that control demands do not modulate task representations. Together, these findings indicate that tasks are represented using a general, context-independent neural code. At first glance, this finding might be taken to imply that these brain regions do not support flexible adaptation of behavior because they do not change flexibly under varying environmental conditions. It has been argued previously that frontal and parietal brain regions support flexible adaptation through flexible task representations that change under varying external demands (Duncan, 2001; Waskom et al., 2014; Woolgar et al., 2015). However, generalized coding under different conditions might also support adaptive behavior: invariant coding allows robust access to task information even if we are confronted with novel situations. This might enable fast transfer of abstract rules (Cole et al., 2011) and stable selective attention toward task-relevant information (Zhang et al., 2013). Stable task representations have also been observed under varying attentional loads (Chan et al., 2015), further highlighting the context-independent coding of tasks. Therefore, our findings of such invariant neural represen- 
tations do not rule out a dynamic adjustment of task-specific neurons, as the adaptive coding hypothesis suggests (Duncan, 2001, 2010; Waskom et al., 2014). Flexible top-down signals may be reflected in different levels of task processing that merely access the robust context-independent representation without modulating it. In addition, we found no significant results in the analysis testing for context-dependent task coding. Although we used a highly sensitive ROI approach, this null finding cannot rule out in principle that there might also be neurons that do code tasks differently for different cognitive control demands.

\section{Role of task switch processes}

Although this study focused on differences and generalizations of neural task representations during switching, we also observed behavioral switch costs. Our paradigm was not designed to determine the source of the underlying processes, but switch costs might arise for a number of reasons, including proactive interference due to task set inertia (Allport et al., 1994), the inhibition of previously executed task sets (Goschke, 2000; Mayr and Keele, 2000), and processes of rule retrieval (goal setting) and rule implementation (Rubinstein et al., 2001). Models of task switching that assume that part of the switch cost reflects proactive interference from previous and/or crosstalk from concurrently active but currently irrelevant task sets would presumably result in task representations that are degraded and less distinct on switch compared with repeat trials. Such an effect should show up in a reduced accuracy with which task representation can be decoded from spatial patterns of brain activity. However, the present findings of task representations that are independent of current switch demands do not suggest such a modulation from whichever source. Neurons in the frontoparietal cortex are able to encode tasks context invariantly under various different conditions such as high- and low-control demands (see also Wisniewski et al., 2016). Switch costs might then arise at a different stage, when task information from the parietal cortex is further processed by brain regions more closely associated with implementing cognitive control (Badre, 2008).

\section{Conclusion}

In summary, our results provide novel insights into the effects of task switching on the distributed neuronal representations of tasks. We did not find reliable differences in task coding between switch and repeat trials. However, task representations in bilateral parietal and left PFC generalized under conditions of high- and low-cognitive-control demands. These results provide further insight into the important function of the frontoparietal network for task representation. Control-independent task coding might enable robust access to task-relevant information under different environmental conditions to support flexible adjustment of behavior.

\section{Notes}

Supplemental material for this article is available at http://neurovault. org/collections/2011/. Included are unthresholded group-level wholebrain maps (spmT) that are presented in the manuscript. This material has not been peer reviewed.

\section{References}

Allport A, Styles EA, Hsieh S (1994) Shifting attentional set: exploring the dynamic control of tasks. Attention and Performance 15:421-452.

Altmann EM (2006) Task switching is not cue switching. Psychonomic Bulletin and Review 13:1016-1022. CrossRef Medline

Altmann EM (2007) Cue-independent task-specific representations in task switching: evidence from backward inhibition. Journal of Experimental
Psychology. Learning, Memory, and Cognition 33:892-899. CrossRef Medline

Arbuthnott KD (2008) Asymmetric switch cost and backward inhibition: carryover activation and inhibition in switching between tasks of unequal difficulty. Canadian Journal of Experimental Psychology 62:91-100. CrossRef Medline

Badre D (2008) Cognitive control, hierarchy, and the rostro: caudal organization of the frontal lobes. Trends Cogn Sci 12:193-200. CrossRef Medline

Bode S, Haynes JD (2009) Decoding sequential stages of task preparation in the human brain. Neuroimage 45:606-613. CrossRef Medline

Brass M, Derrfuss J, Forstmann B, von Cramon DY (2005) The role of the inferior frontal junction area in cognitive control. Trends Cogn Sci 9:314316. CrossRef Medline

Braver TS, Reynolds JR, Donaldson DI (2003) Neural mechanisms of transient and sustained cognitive control during task switching. Neuron 39: 713-726. CrossRef Medline

Bunge SA, Kahn I, Wallis JD, Miller EK, Wagner AD (2003) Neural circuits subserving the retrieval and maintenance of abstract rules. J Neurophysiol 90:3419-3428. CrossRef Medline

Cavina-Pratesi C, Valyear KF, Culham JC, Köhler S, Obhi SS, Marzi CA, Goodale MA (2006) Dissociating arbitrary stimulus-response mapping from movement planning during preparatory period: evidence from event-related functional magnetic resonance imaging. J Neurosci 26: 2704-2713. CrossRef Medline

Chan JL, Kucyi A, DeSouza JF (2015) Stable task representations under attentional load revealed with multivariate pattern analysis of human brain activity. J Cogn Neurosci 27:1789-1800. CrossRef Medline

Cole MW, Etzel JA, Zacks JM, Schneider W, Braver TS (2011) Rapid transfer of abstract rules to novel contexts in human lateral prefrontal cortex. Front Hum Neurosci 5:142. CrossRef Medline

Crone EA, Wendelken C, Donohue SE, Bunge SA (2006) Neural evidence for dissociable components of task-switching. Cereb Cortex 16:475-486. Medline

Dienes Z (2014) Using Bayes to get the most out of non-significant results. Front Psychol 5:781. CrossRef Medline

Dove A, Pollmann S, Schubert T, Wiggins CJ, von Cramon DY (2000) Prefrontal cortex activation in task switching: an event-related fMRI study. Brain Res Cogn Brain Res 9:103-109. CrossRef Medline

Dudschig C, Jentzsch I (2009) Speeding before and slowing after errors: is it all just strategy? Brain Res 1296:56-62. CrossRef Medline

Duncan J (2001) An adaptive coding model of neural function in prefrontal cortex. Nat Rev Neurosci 2:820-829. CrossRef Medline

Duncan J (2010) The multiple-demand (MD) system of the primate brain: mental programs for intelligent behaviour. Trends Cogn Sci 14:172-179. CrossRef Medline

Duncan J (2013) The structure of cognition: attentional episodes in mind and brain. Neuron 80:35-50. CrossRef Medline

Eklund, A., Nichols, T. E., and Knutsson, H (2016) Cluster failure: why fMRI inferences for spatial extent have inflated false-positive rates. Proc Natl Acad Sci U S A 201602413.

Esterman M, Tamber-Rosenau BJ, Chiu YC, Yantis S (2010) Avoiding nonindependence in $\mathrm{fMRI}$ data analysis: Leave one subject out. Neuroimage 50:572-576. CrossRef Medline

Etzel JA, Cole MW, Zacks JM, Kay KN, Braver TS (2016) Reward motivation enhances task coding in frontoparietal cortex. Cereb Cortex 26:16471659. CrossRef Medline

Friston KJ, Jezzard P, Turner R (1994) Analysis of functional MRI timeseries. Hum Brain Mapp 1:153-171. CrossRef

Gilbert SJ (2011) Decoding the content of delayed intentions. J Neurosci 31:2888-2894. CrossRef Medline

Gorgolewski KJ, Varoquaux G, Rivera G, Schwartz Y, Sochat VV, Ghosh SS, Maumet C, Nichols TE, Poline JB, Yarkoni T, Margulies DS, Poldrack RA (2016) NeuroVault.org: A repository for sharing unthresholded statistical maps, parcellations, and atlases of the human brain. Neuroimage 124: 1242-1244. CrossRef Medline

Goschke, T (2000) Intentional reconfiguration and involuntary persistence in task set switching. In: Attention and performance XVIII: control of cognitive processes (Monsell S, Driver J, eds), pp. 331-335. Cambridge, MA: MIT.

Gruber O, Karch S, Schlueter EK, Falkai P, Goschke T (2006) Neural mech- 
anisms of advance preparation in task switching. Neuroimage 31:887895. CrossRef Medline

Haynes JD (2015) A primer on pattern-based approaches to fMRI: principles, pitfalls, and perspectives. Neuron 87:257-270. CrossRef Medline

Haynes JD, Rees G (2006) Decoding mental states from brain activity in humans. Nat Rev Neurosci 7:523-534. CrossRef Medline

Hebart MN, Görgen K, Haynes JD (2016) The Decoding Toolbox (TDT): a versatile software package for multivariate analyses of functional imaging data. Front Neuroinform 8:88. CrossRef Medline

Jamadar S, Hughes M, Fulham WR, Michie PT, Karayanidis F (2010) Neuroimage The spatial and temporal dynamics of anticipatory preparation and response inhibition in task-switching. Neuroimage 51:432-449. CrossRef Medline

Jeffreys H (1961) The theory of probability, ed 3. Oxford: OUP.

Jersild AT (1927) Mental Set and Shift. Archives of Psychology 89:12-47. Available from: https://archive.org/details/mentalsetshift00jers. Accessed August 19, 2016.

Jimura K, Cazalis F, Stover ER, Poldrack RA (2014) The neural basis of task switching changes with skill acquisition. Front Hum Neurosci 8:339. CrossRef Medline

Kaplan JT, Man K, Greening SG (2015) Multivariate cross-classification: applying machine learning techniques to characterize abstraction in neural representations. Front Hum Neurosci 9:151. CrossRef Medline

Kiesel A, Steinhauser M, Wendt M, Falkenstein M, Jost K, Philipp AM, Koch I (2010) Control and interference in task switching-a review. Psychol Bull 136:849-874. CrossRef Medline

Kok A, Ridderinkhof KR, Ullsperger M (2006) The control of attention and actions: current research and future developments. Brain Res 1105:1-6. CrossRef Medline

Kriegeskorte N (2011) Pattern-information analysis: From stimulus decoding to computational-model testing. Neuroimage 56:411-421. CrossRef Medline

Kriegeskorte N, Goebel R, Bandettini P (2006) Information-based functional brain mapping. Proc Natl Acad Sci U S A 103:3863-3868. CrossRef Medline

Kriegeskorte N, Simmons WK, Bellgowan PS, Baker CI (2009) Circular analysis in systems neuroscience: the dangers of double dipping. Nat Neurosci 12:535-540. CrossRef Medline

Liu T, Hou Y (2013) A hierarchy of attentional priority signals in human frontoparietal cortex. J Neurosci 33:16606-16616. CrossRef Medline

Mayr U, Keele SW (2000) Changing internal constraints on action: the role of backward inhibition. Journal of Experimental Psychology: General 129:4-26. CrossRef

Mayr U, Kliegl R (2003) Differential effects of cue changes and task changes on task set selection costs. Journal of Experimental Psychology. Learning, Memory, and Cognition 29:362-372. CrossRef Medline

Meiran, N (2010) Task switching: Mechanisms underlying rigid vs. flexible self-control. In: Task switching: Mechanisms underlying rigid vs. flexible self-control, Ed 1 (Hassin R, Ochsner K, Trope Y, eds), pp. 203-220. New York: OUP.

Mertens G, De Houwer J (2016) Potentiation of the startle reflex is in line with contingency reversal instructions rather than the conditioning history. Biol Psychol 113:91-99. CrossRef Medline

Momennejad I, Haynes JD (2013) Encoding of prospective tasks in the human prefrontal cortex under varying task loads. J Neurosci 33:1734217349. CrossRef Medline

Monsell S (2003) Task switching. Trends Cogn Sci 7:134-140. CrossRef Medline

Norman KA, Polyn SM, Detre GJ, Haxby JV (2006) Beyond mind-reading: multi-voxel pattern analysis of fMRI data. Trends Cogn Sci 10:424-430. CrossRef Medline

Reverberi C, Görgen K, Haynes JD (2012a) Compositionality of rule representations in human prefrontal cortex. Cereb Cortex 22:1237-1246. CrossRef Medline
Reverberi C, Görgen K, Haynes JD (2012b) Distributed representations of rule identity and rule order in human frontal cortex and striatum. J Neurosci 32:17420-17430. CrossRef Medline

Rogers RD, Monsell S (1995) Costs of a predictable switch between simple cognitive tasks. Journal of Experimental Psychology: General 124:207231. CrossRef

Rouder JN, Speckman PL, Sun D, Morey RD, Iverson G (2009) Bayesian $t$ tests for accepting and rejecting the null hypothesis. Psychonomic Bulletin and Review 16:225-237. CrossRef Medline

Rubinstein JS, Meyer DE, Evans JE (2001) Executive control of cognitive processes in task switching. Journal of Experimental Psychology: Human Perception and Performance 27:763-797. CrossRef Medline

Ruge H, Jamadar S, Zimmermann U, Karayanidis F (2013) The many faces of preparatory control in task switching: reviewing a decade of fMRI research. Hum Brain Mapp 34:12-35. CrossRef Medline

Sakai K, Passingham RE (2003) Prefrontal interactions reflect future task operations. Nat Neurosci 6:75-81. Medline

Sohn MH, Ursu S, Anderson JR, Stenger VA, Carter CS (2000) The role of prefrontal cortex and posterior parietal cortex in task switching. Proc Natl Acad Sci U S A 97:13448-13453. CrossRef Medline

Spector A, Biederman I (1976) Mental set and mental shift revisited. American Journal of Psychology 89:669-679. CrossRef

Todd MT, Nystrom LE, Cohen JD (2013) Confounds in multivariate pattern analysis: theory and rule representation case study. Neuroimage 77: 157-165. CrossRef Medline

Vandierendonck A, Liefooghe B, Verbruggen F (2010) Task switching: Interplay of reconfiguration and interference control. Psychol Bull 136:601626. CrossRef Medline

Wagenmakers EJ (2007) A practical solution to the pervasive problems of $\mathrm{p}$ values. Psychonomic Bulletin and Review 14:779-804. CrossRef Medline

Waskom ML, Kumaran D, Gordon AM, Rissman J, Wagner AD (2014) Frontoparietal representations of task context support the flexible control of goal-directed cognition. J Neurosci 34:10743-10755. CrossRef Medline

Wendelken C, Munakata Y, Baym C, Souza M, Bunge SA (2012) Flexible rule use: common neural substrates in children and adults. Developmental Cognitive Neuroscience 2:329-339. CrossRef Medline

Wisniewski D, Reverberi C, Momennejad I, Kahnt T, Haynes JD (2015) The role of the parietal cortex in the representation of task: reward associations. J Neurosci 35:12355-12365. CrossRef Medline

Wisniewski D, Reverberi C, Tusche A, Haynes JD (2015) The neural representation of voluntary task set selection in dynamic environments. Cereb Cortex 25:4715-4726. CrossRef Medline

Wisniewski D, Goschke T, Haynes JD (2016) Similar coding of freely chosen and externally cued intentions in a fronto-parietal network. Neuroimage 134:450-458. CrossRef Medline

Woolgar A, Hampshire A, Thompson R, Duncan J (2011) Adaptive coding of task-relevant information in human frontoparietal cortex. J Neurosci 31:14592-14599. CrossRef Medline

Woolgar A, Thompson R, Bor D, Duncan J (2011) Multi-voxel coding of stimuli, rules, and responses in human frontoparietal cortex. Neuroimage 56:744-752. CrossRef Medline

Woolgar A, Golland P, Bode S (2014) Coping with confounds in multivoxel pattern analysis: What should we do about reaction time differences? A comment on Todd, Nystrom \& Cohen 2013. Neuroimage 98:506-512. CrossRef Medline

Woolgar A, Afshar S, Williams MA, Rich AN (2015) Flexible coding of task rules in frontoparietal cortex: an adaptive system for flexible cognitive control. J Cogn Neurosci 27:1895-1911. CrossRef Medline

Yeung N, Nystrom LE, Aronson JA, Cohen JD (2006) Between-task competition and cognitive control in task switching. J Neurosci 26:1429-1438. CrossRef Medline

Zhang J, Kriegeskorte N, Carlin JD, Rowe JB (2013) Choosing the rules: distinct and overlapping frontoparietal representations of task rules for perceptual decisions. J Neurosci 33:11852-11862. CrossRef Medline 